\section{Targeting the human TREX complex to prevent herpesvirus replication: what is new?}
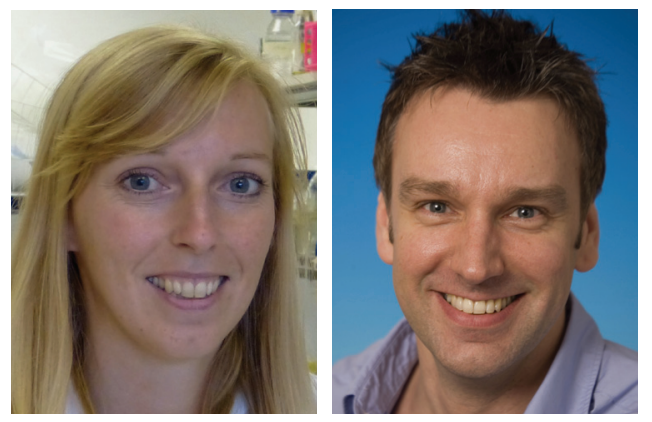

\author{
"We therefore speculated that \\ a small molecule inhibitor \\ disrupting UAP56-mediated \\ ATP hydrolysis would allow an \\ assembly of endogenous \\ hTREX, but prevent KSHV \\ ORF57 recruitment."
}

Sophie Schumann*,1 \& Adrian Whitehouse ${ }^{1}$

First draft submitted: 6 January 2017; Accepted for publication: 12 January 2017; Published online: 24 February 2017

Herpesviruses are a leading cause of human viral disease. There are eight distinct herpesviruses known to cause a range of debilitating acute and recurrent diseases in humans [1-3]. Examples include HSV-1, which causes cold sores and genital lesions; hCMV, which is associated with glandular fever and a range of conditions in immunocompromised patients and congenitally infected newborns; and Kaposi's sarcoma-associated herpesvirus (KSHV), which is an oncogenic herpesvirus associated with commonly fatal malignancies in immunocompromised individuals. Currently, drugs in clinical use for the treatment of herpesvirus infections are inhibitors of herpesvirus DNA polymerases. These nucleotide, nucleoside and pyrophosphate analogs are effective against a number of herpesviruses, although drug-resistant strains are emerging in immunocompromised patients [4]. Moreover, poor efficacy has been reported against the oncogenic herpesvirus subfamily [5]. Consequently, there is an urgent need for the continued development of antiherpesvirus drugs, particularly targeting oncogenic herpesviruses.

Herpesviruses are among the most common viruses found in humans. Once an individual has become infected by a herpesvirus the infection persists, leading to recurrent outbreaks of disease. Herpesviruses have a unique biphasic life cycle, comprising the persistent dormant infection known as the latent state, which can be spontaneously reactivated to initiate the lytic replication cycle. During lytic replication infectious virions are produced, which, in the majority of cases, spread the virus infection, leading to the characteristic disease associated with the herpesvirus. Notably, inhibiting the lytic stages of the herpesvirus life cycle is a key goal for efficacious herpesvirus-targeted therapeutics. Such inhibition, while not eradicating the latent lifelong infection, will treat the pathogenic symptoms associated with herpesvirus infection.

Like all viruses, herpesviruses manipulate and utilize the host cell's machinery to enhance their own replication to produce

\section{KEYWORDS}

- antiviral drugs $\bullet$ herpesviruses

- virology

'School of Molecular \& Cellular Biology, \& Astbury Centre for Structural Molecular Biology, University of Leeds, 
infectious virions, resulting in virus spread and disease. One such example, essential for herpesvirus replication, is the interaction of a conserved family of herpesvirus-encoded RNA-binding proteins with a large cellular multiprotein complex termed TREX [6-10]. Human herpesviruses utilize multiple components of hTREX to stabilize and thus preferentially promote nuclear export of herpesviral mRNAs [11]. Specifically, hTREX serves as a binding platform for the cellular mRNA export factor Nxf1 on herpesvirus mRNAs forming a stable and export competent viral ribonucleoprotein particle.

The recent discovery that a core component of hTREX, the RNA helicase UAP56, remodels the hTREX complex in an ATP-dependent manner $[12,13]$ has given rise to the idea that the interaction of hTREX with herpesvirusencoded adapter proteins might also be regulated in an ATP-dependent manner. This strategy provided an especially attractive option for inhibition of herpesvirus replication, as targeting of the ATPase function of viral and cellular RNA helicases has already been explored, showing selective pharmacological targeting is possible [14-16]. Notably, the targeting of a cellular RNA helicase would also reduce the risk of viral resistance and would have potential pan-herpesvirus activity.

In a recent publication, the novel approach of targeting hTREX to prevent herpesvirus lytic replication is demonstrated [17]. Using co-immunoprecipitation assays we first identified that ATP-dependent remodeling of hTREX affects the interaction of the KSHVencoded adapter protein, known as ORF57, independently of the native hTREX complex. Specifically, formation of the complete hTREX required binding of ATP by UAP 56. However, this ATP-bound state was insufficient to recruit the KSHV ORF57 protein. Here, a further ATP hydrolysis event was essential to mediate the ORF57-hTREX interaction. We therefore speculated that a small molecule inhibitor disrupting UAP56-mediated ATP hydrolysis would allow an assembly of endogenous hTREX, but prevent KSHV ORF57 recruitment. This, in turn, would prevent herpesvirus viral ribonucleoprotein particle formation and subsequently viral lytic replication. Importantly, as hTREX recruitment is not specific to KSHV but conserved in other human herpesviruses, any inhibitory compound targeting the activity of the cellular UAP56 protein should also inhibit lytic replication in a potentially pan-herpesvirus fashion.

A virtual high-throughput screening approach was therefore used to identify potential inhibitors of UAP56 ATPase activity. Following in vitro screening, the prioritized hit compound, CCT018159, was shown to specifically disrupt the interaction of multiple herpesviral adapter proteins, KSHV ORF57, HSV-1 ICP27 and hCMV pUL69, with UAP56, without disrupting endogenous hTREX assembly. Furthermore, the compound inhibited KSHV viral mRNA, but not cellular bulk mRNA nuclear export. Moreover, CCT018159 relieved cells from ORF57-imposed hTREX sequestration, resulting in reduced KSHV DNA replication and infectious virion production. Similar inhibition was also observed for HSV-1 and hCMV lytic replication and infectious virion production.

Our results suggest that a clear therapeutic window, which inhibits herpesvirus replication with limited host cell toxicity, can be achieved. However, CCT018159 is also a known HSP90 inhibitor [18], and although we could effectively inhibit UAP56 activity within HSP90 functional parameters, CCT018159-mediated inhibition of HSP90 at higher concentrations restricted the therapeutic window. This highlights the need for further generation of a more targeted UAP56 inhibitory compound with reduced off-target effects. Here, future medicinal chemistry approaches will endeavor to increase the affinity of CCT018159-related compounds for UAP56, as well as improve its complex pharmacokinetics, which include a relatively high metabolic turnover, and reduce the above-mentioned HSP90-related off-target effects. However, the identification and subsequent characterization of CCT018159 against KSHV, HSV-1 and hCMV has shown that it is possible to target the hTREX complex to inhibit herpesvirus lytic replication. Although we are still several generations from a feasible drug in clinical use, this can be seen as a starting point for lead compound development.

One obvious limitation of this novel targeting approach to treat herpesvirus-related diseases includes, for example, EBV latency-associated lymphomas and carcinomas, and other diseases associated with herpesvirus latent gene expression. Interestingly, however, UAP56 has also been implicated in mRNA processing of other distinct groups of viruses, such as influenza $A$ virus and $\mathrm{HBV}$. While influenza $A$ virus is 
believed to require a virally encoded adapter protein to bridge the interaction between viral mRNA and hTREX [19], HBV employs a special cellular adapter protein [20]. This allows for the intriguing idea that targeting hTREX ATPdependent remodeling may also be effective at inhibiting not only herpesviruses, but other distinct classes of viruses.

In summary, our study has highlighted a novel approach to target herpesvirus lytic replication and potentially other human pathogens through inhibition of the hTREX complex. While the identified inhibitor is only a hit compound, which now requires a lengthy hit-to-lead development process to produce a compound fit for clinical use, the findings are extremely encouraging, as they demonstrate movement toward a new generation of antiherpesvirus drugs.

\section{Open access}

This article is distributed under the terms of the Creative Commons Attribution License 4.0 which permits any use, distribution, and reproduction in any medium, provided the original author(s) and the source are credited. To view a copy of the license, visit http://creativecommons.org/ licenses/by/4.0/

\section{Financial \& competing interests disclosure}

This work was supported in part by the Wellcome Trust (093788/Z/10/Z), Worldwide Cancer Research (121045), BBSRC (BB/000306; BB/M006557) and CRUK (C12057/A19430). The authors have no other relevant affiliations or financial involvement with any organization or entity with a financial interest in or financial conflict with the subject matter or materials discussed in the manuscript apart from those disclosed.

No writing assistance was utilized in the production of this manuscript.

\author{
“This allows for the \\ intriguing idea that \\ targeting hTREX ATP- \\ dependent remodeling \\ may also be effective at \\ inhibiting not only \\ herpesviruses, but other \\ distinct classes of viruses."
}

\section{References}

1 Gilden DH, Mahalingam R, Cohrs RJ, Tyler KL. Herpesvirus infections of the nervous system. Nat. Clin. Pract. Neurol. 3(2), 82-94 (2007).

2 Owen CB, Hughes DJ, Baquero-Perez B et al. Utilising proteomic approaches to understand oncogenic human herpesviruses (Review). Mol. Clin. Oncol. 2(6), 891-903 (2014).

3 Quadrelli C, Barozzi P, Riva G et al. $\beta$-HHVs and HHV-8 in lymphoproliferative disorders. Mediterr. J. Hematol. Infect. Dis. 3(1), e2011043 (2011).

4 Bacon TH, Levin MJ, Leary JJ, Sarisky RT, Sutton D. Herpes simplex virus resistance to acyclovir and penciclovir after two decades of antiviral therapy. Clin. Microbiol. Rev. 16(1), 114-128 (2003).

5 Fife K, Gill J, Bourboulia D, Gazzard B, Nelson M, Bower M. Cidofovir for the treatment of Kaposi's sarcoma in an HIV-negative homosexual man. Br. J. Dermatol. 141(6), 1148-1150 (1999).

6 Koffa MD, Clements JB, Izaurralde E et al. Herpes simplex virus ICP27 protein provides viral mRNAs with access to the cellular mRNA export pathway. EMBO J. 20 (20), 5769-5778 (2001).

7 Lischka P, Toth Z, Thomas M, Mueller R, Stamminger T. The UL69 transactivator protein of human cytomegalovirus interacts with DEXD/H-box RNA helicase UAP56 to promote cytoplasmic accumulation of unspliced RNA. Mol. Cell. Biol. 26(5), 1631-1643 (2006).

8 Semmes OJ, Chen L, Sarisky RT, Gao Z, Zhong L, Hayward SD. Mta has properties of an RNA export protein and increases cytoplasmic accumulation of Epstein-Barr virus replication gene mRNA. J. Virol. 72(12), 9526-9534 (1998).

9 Ote I, Lebrun M, Vandevenne P et al. Varicella-Zoster virus IE4 protein interacts with SR proteins and exports mRNAs through the TAP/NXF1 pathway. PLoS ONE 4(11), e7882 (2009).

10 Jackson BR, Boyne JR, Noerenberg M et al. An interaction between KSHV ORF57 and UIF provides mRNA-adaptor redundancy in herpesvirus intronless mRNA export. PLoS Pathog. 7(7), e1002138 (2011).

11 Schumann S, Jackson B, Baquero-Perez B, Whitehouse A. Kaposi's sarcoma-associated herpesvirus ORF57 protein: exploiting all stages of viral mRNA processing. Viruses 5(8), 1901-1923 (2013).

12 Dufu K, Livingstone MJ, Seebacher J, Gygi SP, Wilson SA, Reed R. ATP is required for interactions between UAP56 and two conserved mRNA export proteins, Aly and CIP29, to assemble the TREX complex. Genes Dev. 24(18), 2043-2053 (2010).

13 Chi B, Wang Q, Wu G et al. Aly and THO are required for assembly of the human TREX complex and association of TREX components with the spliced mRNA. Nucleic Acids Res. 41(2), 1294-1306 (2013).
14 Tsumuraya T, Ishikawa C, Machijima Y et al. Effects of hippuristanol, an inhibitor of eIF4A, on adult T-cell leukemia. Biochem. Pharmacol. 81(6), 713-722 (2011).

15 Yedavalli VSRK, Zhang N, Cai H et al. Ring expanded nucleoside analogues inhibit RNA helicase and intracellular human immunodeficiency virus type 1 replication. J. Med. Chem. 51(16), 5043-5051 (2008).

16 Lindqvist L, Oberer M, Reibarkh M et al. Selective pharmacological targeting of a DEAD box RNA helicase. PLoS ONE 3(2), e1583 (2008).

17 Schumann S, Jackson BR, Yule I et al. Targeting the ATP-dependent formation of herpesvirus ribonucleoprotein particle assembly as an antiviral approach. Nat. Microbiol. 2, 16201 (2016).

18 Dymock BW, Barril X, Brough PA et al. Novel, potent small-molecule inhibitors of the molecular chaperone Hsp90 discovered through structure-based design. J. Med. Chem. 48(13), 4212-4215 (2005).

19 Balasubramaniam VRMT, Wai TH, Tejo BA, Omar AR, Hassan SS. Highly pathogenic avian influenza virus nucleoprotein interacts with TREX complex adaptor protein Aly/ REF. PLoS ONE 8(9), e72429 (2013).

20 Chi B, Wang K, Du Y et al. A sub-element in PRE enhances nuclear export of intronless mRNAs by recruiting the TREX complex via ZC3H18. Nucleic Acids Res. 42(11), 7305-7318 (2014). 